\title{
Article
}

Subscriber access provided by Universidad de Alicante

\section{Time-Resolved Dynamics of Intracrystalline Mesoporosity Generation in Zeolite USY}

Aseem Chawla, Noemi Linares, Jeffrey D Rimer, and Javier García-Martínez

Chem. Mater., Just Accepted Manuscript • DOI: 10.1021/acs.chemmater.9b00435 • Publication Date (Web): 03 Apr 2019

Downloaded from http://pubs.acs.org on April 12, 2019

\section{Just Accepted}

"Just Accepted" manuscripts have been peer-reviewed and accepted for publication. They are posted online prior to technical editing, formatting for publication and author proofing. The American Chemical Society provides "Just Accepted" as a service to the research community to expedite the dissemination of scientific material as soon as possible after acceptance. "Just Accepted" manuscripts appear in full in PDF format accompanied by an HTML abstract. "Just Accepted" manuscripts have been fully peer reviewed, but should not be considered the official version of record. They are citable by the Digital Object Identifier (DOI®). "Just Accepted" is an optional service offered to authors. Therefore, the "Just Accepted" Web site may not include all articles that will be published in the journal. After a manuscript is technically edited and formatted, it will be removed from the "Just Accepted" Web site and published as an ASAP article. Note that technical editing may introduce minor changes to the manuscript text and/or graphics which could affect content, and all legal disclaimers and ethical guidelines that apply to the journal pertain. ACS cannot be held responsible for errors or consequences arising from the use of information contained in these "Just Accepted" manuscripts. 


\title{
Time-Resolved Dynamics of Intracrystalline Mesoporosity Generation in Zeolite USY
}

\author{
Aseem Chawla, Noemi Linares, Jeffrey D. Rimer*, Javier García-Martínez*
}

\author{
A. Chawla, Prof. J.D. Rimer, Department of Chemical and Biomolecular Engineering, University of \\ Houston, Houston, TX 77204, USA \\ Dr. N. Linares, Prof. J. García-Martínez, Molecular Nanotechnology Lab, Department of Inorganic \\ Chemistry, University of Alicante, 03690 Alicante, Spain \\ * Email: jrimer@central.uh.edu and j.garcia@ua.es
}

\begin{abstract}
The treatment of zeolites with surfactants in alkaline media is an effective and versatile technique to impart intracrystalline well-defined mesoporosity in these materials. In this study, the dynamics of surface reconstruction that occurs during the treatment of USY zeolite by surfactant-templating was monitored in situ by atomic force microscopy. The development of surfactant-templated mesoporosity and the concurrent healing of defects that are characteristic of steamed zeolites occur in less than one hour at room temperature, which emphasizes the low energy barriers needed to reorganize the crystalline structure of this zeolite. This transformation was also followed by X-ray diffraction, $\mathrm{N}_{2}$ adsorption, and TEM analysis of ultramicrotomed samples to confirm that the rapid formation of surfactant-templated mesoporosity and the reconstruction of the zeolite crystals occur not only on the surface of the zeolite, but homogeneously throughout the whole zeolite. This process involves a significant and rapid breaking and re-formation of bonds; however, the zeolite does not dissolve during this process as solids recovery at any given time of the treatment is approximately $100 \%$ and the concentration of soluble $\mathrm{Si}$ or Al species in the liquid is negligible. Parametric analysis revealed that excessive $\mathrm{NaOH}$ leads to the partial transformation of zeolite into an amorphous mesoporous solid,
\end{abstract}


while insufficient quantity of base and/or treatment time can lead to an incomplete mesostructuring of the zeolite, which highlights the importance of judiciously selecting the treatment conditions for every given zeolite.

\section{Table of Contents (TOC) Graphic:}

Time-resolved monitoring of the surfactant-templating process of zeolite USY allowed for the direct observation of the crystal rearrangement to accommodate well-defined mesoporosity while the preexistent broad porosity, produced during processes of dealumination, disappears. The surface dynamics of this process reveals low energy barriers and a high degree of flexibility of this zeolite to reorganize during mesostructuring.

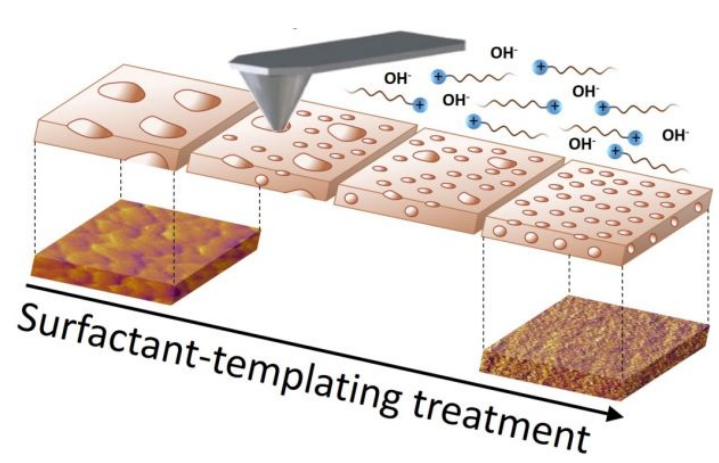




\section{Introduction}

For more than 25 years the surfactant-templating approach has been employed to facilitate the mesostructuration of different materials through supramolecular self-assembly of surfactants. ${ }^{1}$ Since the first publication regarding the surfactant-templating route for mesoporous silica, ${ }^{2}$ the number of studies reporting the preparation of nanostructured mesoporous solids via supramolecular templating has been continuously increasing and expanding to more diverse inorganic, organic/inorganic hybrid, and organic solid materials. ${ }^{3}$ More recently, the surfactant-templating approach has also been extended to the synthesis of hierarchical zeolites in which the surfactant can be used to generate intracrystalline mesoporosity in the structure of these microporous materials. ${ }^{4}$ Among the different procedures developed to generate secondary porosity within zeolites, ${ }^{5-10}$ surfactant-templating in alkaline media allows for the incorporation of mesoporosity with tailored dimensions, while simultaneously maintaining the strong acidity and hydrothermal stability of the original zeolite. ${ }^{11-15}$ The structure of surfactant-templated zeolites has been resolved by a combination of advanced gas adsorption, rotation electron diffraction (RED), and electron tomography (ET), which unambiguously confirmed the presence of intracrystalline mesoporosity within the zeolites. ${ }^{16,} 17$ The simplicity, low cost, and versatility of this procedure to generate hierarchical zeolites has led to its commercialization and use as an FCC catalyst in several refineries to more efficiently produce liquid fuels while reducing $\mathrm{CO}_{2}$ emissions. ${ }^{13}$ The most plausible mechanism for the formation of these surfactant-templated zeolites relies on the crystal reconstruction of the zeolite that occurs through multiple processes: (i) the diffusion of cationic surfactants to the interior of the zeolites attracted by the negatively charged $\equiv \mathrm{Si}$ $\mathrm{O}^{-}$sites formed by the opening of $\mathrm{Si}-\mathrm{O}-\mathrm{Si}$ bonds by hydroxide ions; (ii) the self-assembly of the surfactant molecules into micelles within the zeolite structure; and (iii) the expansion of the zeolite crystals in order to accommodate the formed mesoporosity. ${ }^{18}$ 
Recently, we reported the first time-resolved study of the development of mesoporosity in zeolites through surfactant-templating by in situ synchrotron XRD. ${ }^{19}$ By combining these measurements with a number of ex situ techniques, we were able to determine the apparent activation energy of the development of mesoporosity in USY by surfactant-templating, which is in the same order of magnitude $\left(30-65 \mathrm{~kJ} \mathrm{~mol}^{-1}\right)$ to those involved in the crystallization of zeolites. ${ }^{20}$ Additionally, the use of a time-resolved technique, such as liquid-cell transmission electron microscopy (Liq-TEM), rendered the first in situ real time visualization of this process. ${ }^{19}$ Due to the presence of liquid water and the silicon nitride windows of the TEM sample cell, the incident-wave amplitude was attenuated, reducing the resolution, which hampered the visualization of features below $5 \mathrm{~nm}$. This limitation prevented the visualization of the development of surfactant-templated mesoporosity, which was confirmed posteriori by HR-TEM. Collective experiments revealed two interesting phenomena of this process: the disappearance of the secondary broad mesoporosity $(20-30 \mathrm{~nm})$ of the parent USY zeolite, and the preservation of the crystal, which does not dissolve during the process. ${ }^{19}$

In this study we directly visualized the surface reconstruction of zeolite USY using atomic force microscopy (AFM), which is a widely used technique that is capable of capturing dynamic events of crystal surfaces at near molecular resolution. AFM has proven especially useful for examining the crystallization of zeolites and metal-organic frameworks (MOFs) that include LTA, ${ }^{21} \mathrm{MFI},{ }^{22,}{ }^{23} \mathrm{LTL},{ }^{24}$ SAV,${ }^{25}$ FAU, ${ }^{26}$ CHA,${ }^{27}$ MOF-5, ${ }^{26}$ and HKUST-1, ${ }^{28}$ among others. ${ }^{21-26}$ Rimer and coworkers ${ }^{21}$ recently demonstrated the use of in situ AFM to illustrate the diverse precursors involved in the crystallization of zeolite LTA ranging from monomers and oligomers to nanocrystals and gel-like islands. Weckhuysen and coworkers ${ }^{28}$ employed in situ AFM to elucidate the nucleation and growth mechanisms of MOF thin films; however, to the best of our knowledge, AFM has never been used to study the formation of intracrystalline mesoporosity in zeolites. 
Here, we use AFM to systematically examine the generation of mesoporosity in USY zeolites by surfactant-templating. This technique allows for the in situ visualization of individual crystals to elucidate processes related to the mesostructuring of zeolites, including both the formation of mesoporosity and the disappearance of the broad porosity present in the original USY. Bulk characterization techniques, such as $\mathrm{N}_{2}$ adsorption, ${ }^{27} \mathrm{Al}$ NMR and XRD, were used to confirm the uniformity of mesopores throughout the zeolite (i.e. surface and interior). Ultramicrotomed slices of the zeolite mesostructured at different times were examined by TEM to monitor mesoporosity development throughout the zeolite crystals and to relate these observations with the phenomena studied on the surface by AFM. Collectively, these findings reveal low energy barriers and short timescales for the facile restructuring of zeolite USY.

\section{Experimental Methods}

Materials. USY zeolite (CBV780 with a Si/Al molar ratio of 40 as indicated by the supplier) was purchased from Zeolyst. Hexadecyltrimethylammonium bromide (CTAB) (98\%) was purchased from Sigma-Aldrich. Sodium hydroxide ( $98 \%$ pellets) was supplied by MACRON Fine Chemicals. Deionized (DI) water was prepared using an Aqua Solutions RODI-C-12A purification system (18.2 $\mathrm{M} \Omega)$. All reagents were used as received without further purification.

Preparation of treatment solution. The basic surfactant solution used for in situ AFM experiments was prepared by mixing the required quantity of all components to yield a solution with molar composition $1 \mathrm{NaOH}: 1.08 \mathrm{CTAB}: 888 \mathrm{H}_{2} \mathrm{O} \cdot{ }^{20}$ In order to obtain a $62.5 \mathrm{mM} \mathrm{NaOH}$ solution, $\mathrm{NaOH}(0.20 \mathrm{~g})$ was added to DI water $(80 \mathrm{~g})$ and mixed in a polypropylene (PP) bottle. Thereafter, CTAB (2 g) was added to this mixture and continuously stirred for $15 \mathrm{~min}$ at $40{ }^{\circ} \mathrm{C}$ to obtain a clear solution. The PP bottle was then heated in a Thermo-Fisher Precision Premium 3050 Series gravity 
oven at $90{ }^{\circ} \mathrm{C}$ for 1 day and was quenched to room temperature (RT). Herein, this solution is referred to as $\mathrm{S} 2$.

Scanning probe microscopy. All AFM measurements were performed on an Asylum Research Cypher ES instrument (Santa Barbara, CA) equipped with a liquid sample cell. Zeolite USY crystals were firmly placed on a 15-mm specimen disk (Ted Pella, Inc.) using quickset Loctite epoxy (Henkel Corporation) that was cured in an oven at $50{ }^{\circ} \mathrm{C}$ for $24 \mathrm{~h}$. The sample was then removed from the oven and cooled to room temperature in air. The specimen disk was rinsed with DI water to remove loosely bound crystals, and dried under inert argon gas to remove dust. The sample was then placed in a closed AFM liquid cell and images were collected using a $\mathrm{Cr} / \mathrm{Au}$-coated silicon nitride cantilever (Olympus TR800PSA) with a spring constant of $0.57 \mathrm{~N} \mathrm{~m}^{-1}$. The crystal substrate was first scanned in air to locate a desired imaging area. Solution S2 was then introduced into the AFM cell by a syringe and the system was left to equilibrate at RT for ca. 30 min. During in situ measurements, solution S2 was continuously supplied to the liquid cell using a syringe pump (Razel Scientific Instruments, Model R100-E) at a rate of $2 \mathrm{~cm}^{3} \mathrm{~min}^{-1}$. AFM images were collected in tapping mode to minimize tipsubstrate contact using at a scan rate of $1-8 \mathrm{~Hz}$ and 256 lines per scan.

Ex-situ treatment of samples. The preparation of surfactant-templated mesoporous zeolites ex situ was carried out by first dissolving CTAB $(0.5 \mathrm{~g})$ in $40 \mathrm{~mL}$ of $62.5 \mathrm{mM} \mathrm{NaOH}$ (S2 solution). To this mixture was added $1 \mathrm{~g}$ of CBV 780 zeolite and the resultant slurry was stirred at RT for $1 \mathrm{~min}$. The surfactant-templating process was performed under static conditions by allowing the zeolite to remain in contact with the alkaline surfactant solution for a set amount of time at RT. Solutions of varying $\mathrm{NaOH}$ concentration, ranging from $38 \mathrm{mM}$ (solution S1) to $94 \mathrm{mM}$ (solution S3), were also prepared to evaluate the influence of $\mathrm{pH}$ on the quality of hierarchical zeolites and the kinetics of mesostructuring. Calcination of the samples was carried out under the flow of dry air at $823 \mathrm{~K}$ for $5 \mathrm{~h}\left(2 \mathrm{~K} \mathrm{~min}^{-1}\right)$. 
Ex situ characterization of samples. The morphology of the mesoporous materials was investigated by transmission electron microscopy (TEM) using a JEM-2010 microscope (JEOL, 200 $\mathrm{kV}, 0.14 \mathrm{~nm}$ of resolution). Selected samples were embedded in a Spurr resin, cured, and cut into slices $80 \mathrm{~nm}$ thin using a RMC-MTXL ultramicrotome (Boeckeler Instruments, Tucson, AZ). These slices were then placed on a grid to study the interior of the zeolite crystals by TEM. Digital analysis of TEM micrographs was performed using Gatam DigitalMicrographTM 1.80.70 for GMS 1.8. Porous texture was characterized by $\mathrm{N}_{2}$ gas adsorption/desorption at $77 \mathrm{~K}$ in a Quadrasorb-Kr/MP apparatus from Quantachrome Instruments. The samples were previously degassed for $4 \mathrm{~h}$ at $250{ }^{\circ} \mathrm{C}$ at $5 \times 10^{-5}$ bars. Adsorption data were analyzed using the software QuadraWin (version 6.0) of Quantachrome Instruments. Cumulative pore volumes and pore-size distribution curves were calculated using a density functional theory method (NL-DFT adsorption branch model) from the adsorption branch of the isotherms, as described elsewhere. ${ }^{29}$ From the cumulative pore volume determined by NL-DFT, the micropore volume was obtained at a pore size of $2 \mathrm{~nm}$; the mesopore volume was calculated by subtracting the micropore volume from the pore volume at a pore size of $8 \mathrm{~nm}$ (to discard the large mesoporosity due to the steaming present in the original USY); and the large mesopore volume was obtained by subtracting the micropore and mesopore volume from the total pore volume obtained at a relative pressure $\left(P / P_{0}\right)$ of 0.95 . X-ray diffraction $(\mathrm{XRD})$ patterns were collected on a Seifert XRD 3003 TT (Bragg-Brentano geometry) powder diffractometer using $\mathrm{Cu} \mathrm{K \alpha}$ radiation $(\lambda=1.54056 \AA)$ at a scanning velocity of $0.05^{\circ} \mathrm{min}^{-1}$ in the $5^{\circ}<2 \theta<50^{\circ}$ range. Solid state NMR analyses were carried out in a $500 \mathrm{MHz}$ Bruker Avance III HD Spectrometer using the pulse program ZG. The samples were spun at a rate of $4 \mathrm{kHz}$ and the ${ }^{27} \mathrm{Al} \mathrm{NMR}$ spectra were obtained with a relaxation delay of $5 \mathrm{~s}$ and 2048 scans. Inductively coupled plasma optical emission spectrometry (ICP-OES) analyses of the liquid mixtures after the surfactant-templating treatment were performed using a Perkin Elmer spectrometer (Optima 4300 DV model). 


\section{Results and Discussion}

We used in situ AFM to monitor the evolution of surface features on USY crystals (Zeolyst CBV 780) during mesostructuring. The size of as received USY crystals is $600-800 \mathrm{~nm}$ (Figure S1), which is comparatively smaller than single crystals typically used for AFM analyses. This required a multitiered approach to locate appropriate surfaces for in situ imaging wherein the sample was first imaged with a large scan size (e.g. $5 \times 5 \mathrm{~m}^{2}$ area, Figure S1), followed by a progressive reduction in scan size to encompass a single USY crystal. The imaged surface is often located within an agglomerate of multiple crystals, as shown in Figure 1A. Only surfaces oriented flat relative to the plane of imaging were selected for analysis. The surfaces of USY samples are comprised of protrusions (Figures 1B-D and S2) ranging in height from 1 to $6 \mathrm{~nm}$ and width from 20 to $40 \mathrm{~nm}$ (Figure 1C), noting that the width of protrusions is an overestimate due to the curvature of AFM tips (ca. $20 \mathrm{~nm}$ ). Interestingly, the rough topography of USY crystals is consistent with the defects reported in literature using techniques such as TEM and nitrogen physisorption. ${ }^{19}, 30$ These features are induced by steaming and acid treatments carried out by the supplier to remove aluminum from the framework, thereby converting zeolite HY to USY. ${ }^{30}$
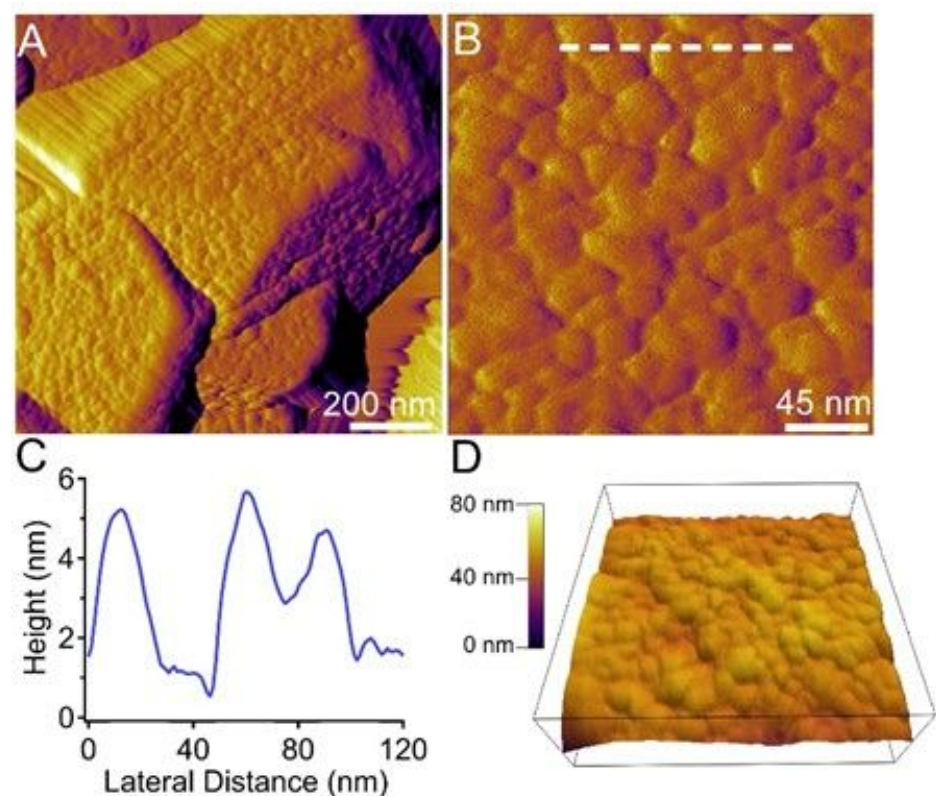
Figure 1. (A and B) AFM images of as received USY (CBV 780, FAU type) in amplitude mode revealing rough surface protrusions. (C) Height profile along the dashed line in panel B showing a representative topography of untreated crystals. (D) 3-dimensional image of the surface depicted in panel B.

Mesostructuring was performed at room temperature using a mild basic solution $(62.5 \mathrm{mM} \mathrm{NaOH})$ containing $68.6 \mathrm{mM}$ surfactant (solution S2). Upon introduction of this solution to the AFM liquid cell, we observed a smoothening of crystal surfaces within the first $30 \mathrm{~min}$ of scanning. This is evident in time-resolved AFM images (Figure 2A - C and movie S1), which show a temporal reduction in the heights of rough features initially present on the crystal surface. Figure $2 \mathrm{C}$ depicts changes in the root mean square (RMS) area roughness of the crystal surface obtained from measurements of multiple 500 $\times 500 \mathrm{~nm}^{2}$ scan areas. The disappearance of the rough islands (convex protrusions), which are one type of USY surface defect, results in the reduction of RMS roughness. It is worth noting that rapid fluctuations in surface features during the first $30 \mathrm{~min}$ of treatment can give a false impression of nonmonotonic smoothening (movie S1). A progressive reduction in surface roughness is more evident at later treatment times, as depicted in movies S2-S5. Tracking pore size before and after exposure to solution S2 reveals a progressive decrease in the average pore diameter (Figure 2D) with a concomitant narrowing of the pore size distribution, as expected due to the ability of the surfactant to direct the formation of uniform mesopores. It is important to note that USY surfaces begin reconstructing and forming mesopores within the first minutes of exposure to the solution, which is qualitatively consistent with $\mathrm{N}_{2}$ physisorption and TEM results (see Figures 6 and 7) showing that large pores in as received USY crystals (i.e. the byproduct of steam treatment) disappear during surfactant treatment. 


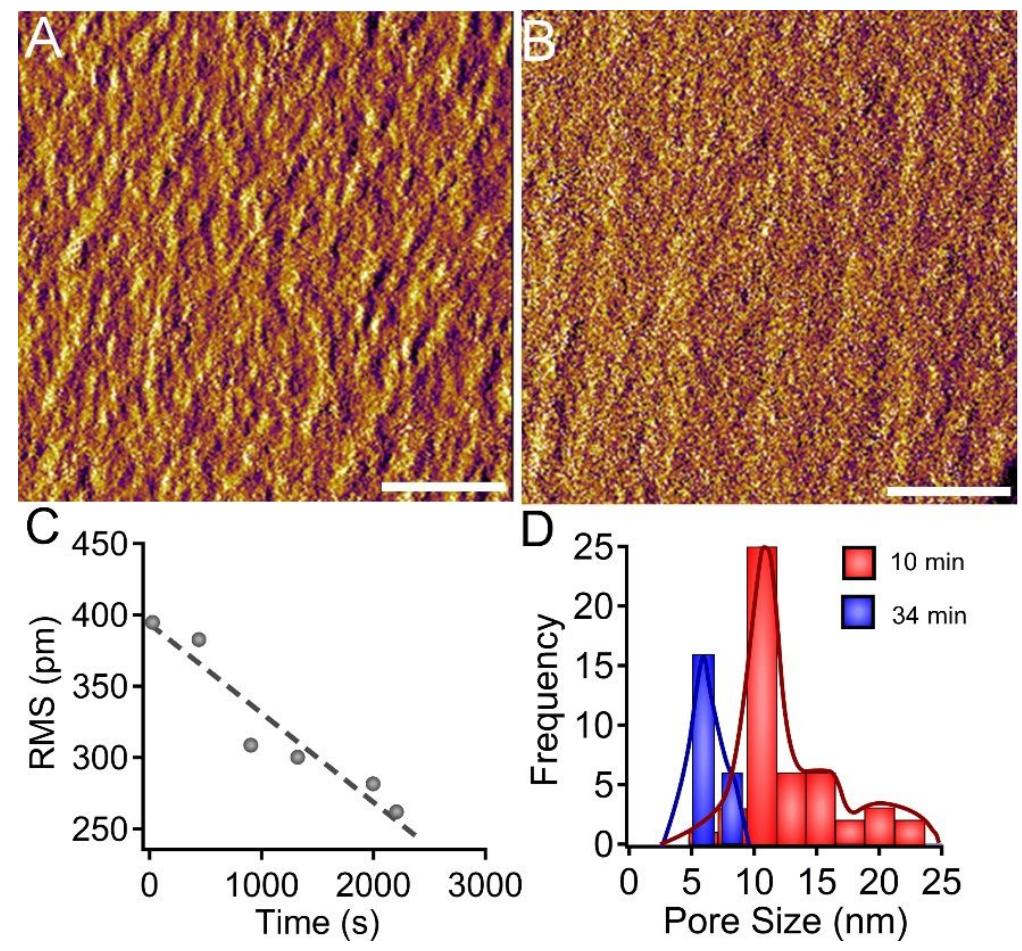

Figure 2. (A and B) Time-resolved in situ AFM amplitude mode images of a USY crystal treated with solution S2 at room temperature showing the disappearance of rough features present on the crystal surface with the concomitant appearance of mesopores. Images correspond to (A) 10 min and (B) 34 min of continuous scanning. Scale bars are equal to $100 \mathrm{~nm}$. (C) Temporal reduction in the root mean square (RMS) roughness of scanned areas $\left(500 \times 500 \mathrm{~nm}^{2}\right)$ indicates a smoothening of surface protrusions. (D) Statistical analysis of mesopore sizes that were measured on at least 40 areas of a single USY sample after $10 \mathrm{~min}$ (red) and $34 \mathrm{~min}$ (blue) of treatment with solution S2.

Here we report a rapid reconstruction of surface defects during mesostructuring. We begin with an analysis of 3-dimensional (3D) islands, or convex protrusions, analogous to those observed in Figures 1 and 2. Exposure of USY crystal surfaces to solution S2 results in a temporal reduction in the height of 3D islands (arrow in Figure 3A) that occurs in parallel with the emergence of new mesopores (callout in Figure 3B). The height profiles along the dashed line in each micrograph of Figure 3 reveal a 
temporal healing of convex protrusions wherein the defect height decreases by a factor of two within 6 min of imaging (Figure 3D). In order to confirm that the disappearance of 3D islands was representative of the entire sample, and not a local effect of the AFM tip, we collected AFM images of different crystal surfaces at periodic times during treatment (Figures S4 and S5). The collection of images corroborated the general trend observed in Figure 3, thus indicating that surface reconstruction results in the progressive disappearance of convex topographies. These observations also confirm that the surface phenomena and underlying crystal reconstruction that occurs during surfactant-templating have low energy barriers, as previously measured by in situ X-ray diffraction and gas adsorption. ${ }^{20}$
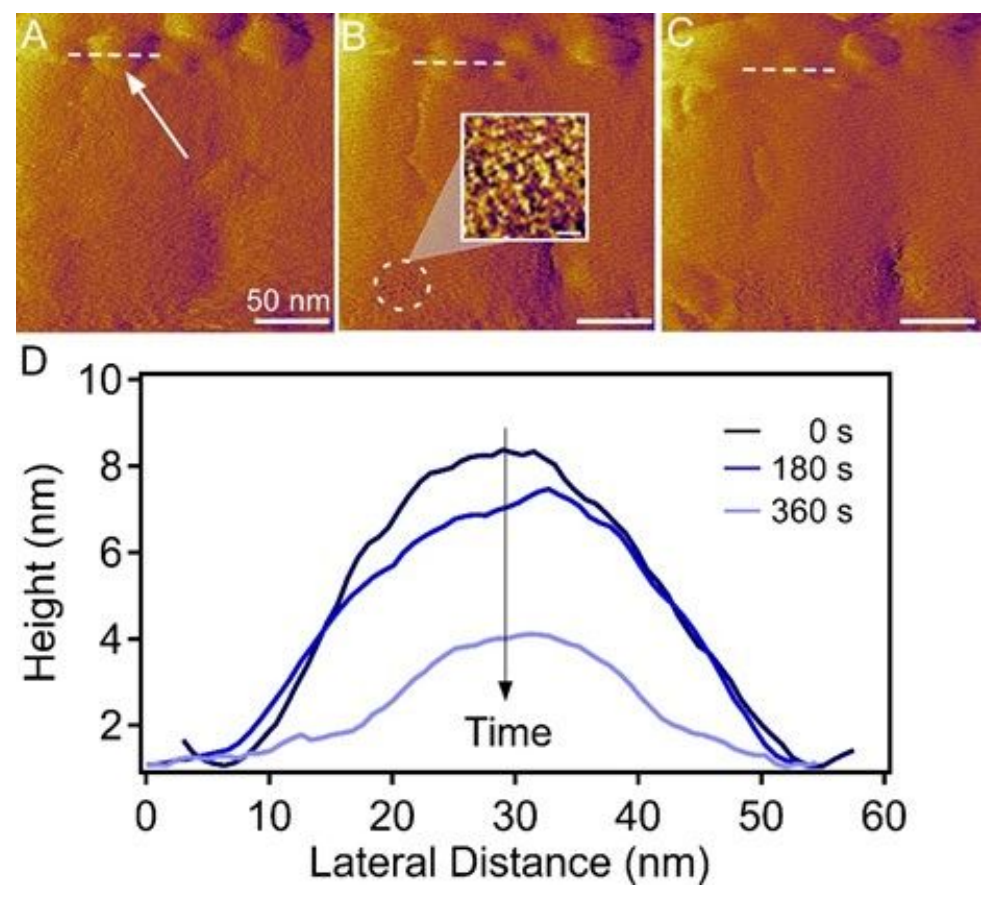

Figure 3. $(\mathrm{A}-\mathrm{C})$ Snapshots from movie S2 showing temporal changes of the same USY crystal surface after $4 \mathrm{~h}$ of treatment in solution $\mathrm{S} 2$ at $25{ }^{\circ} \mathrm{C}$. There is a gradual disappearance of $3 \mathrm{D}$ protrusions (white arrow) and the appearance of mesopores (callout of dashed white circle; scare bar equals $10 \mathrm{~nm}$ ). Images were collected in ca. $180 \mathrm{~s}$ intervals during continuous scanning. (D) Height profiles along the dashed white lines reveal a monotonic decrease in the height of islands (convex protrusions). 
In addition to convex protrusions, AFM measurements reveal the annealing of two additional types of defects: macrosteps and concave pits (Figure 4A). The presence of large steps (ca. $30 \mathrm{~nm}$ height) is evident on several USY samples (Figure 4B and C). Mesostructuring results in the annealing of these surface features to render the interface more level, as indicated in height profiles during periodic imaging times (Figure 4D). We also observe numerous concave cavities (or pits) on USY crystal surfaces (Figures 4E and S3). In situ AFM measurements reveal that the latter features are healed by surfactant treatment and the morphology of pits changes from a circular to ellipsoid shape (Figure 4E and F). Height profiles of a single pit (Figure 4G) reveal that these concave defects become shallower with prolonged exposure to solution S2. Indeed, there is a 4-fold reduction in pit height after 43 min of mesostructuring. To once again confirm that this healing effect caused by the treatment with the surfactant was not attributed to the movement of the AFM tip during continuous imaging, we tracked the evolution of several crystal surfaces (Figures S7 and S8) and observed a similar transition to more level interfaces laden with uniformly-sized mesopores. Low magnification AFM images of USY crystals after a 6-h treatment (Figure S10) further shows that the final USY crystal surface is devoid of any defects. The ability of surfactant to heal the defects of USY crystals is consistent with timeresolved ex situ $\mathrm{N}_{2}$ physisorption data and TEM images (Figures 6 and 7, respectively), and with our previous results for a for a less severely steamed CBV720 USY zeolite as published elsewhere. ${ }^{18,19}$ 

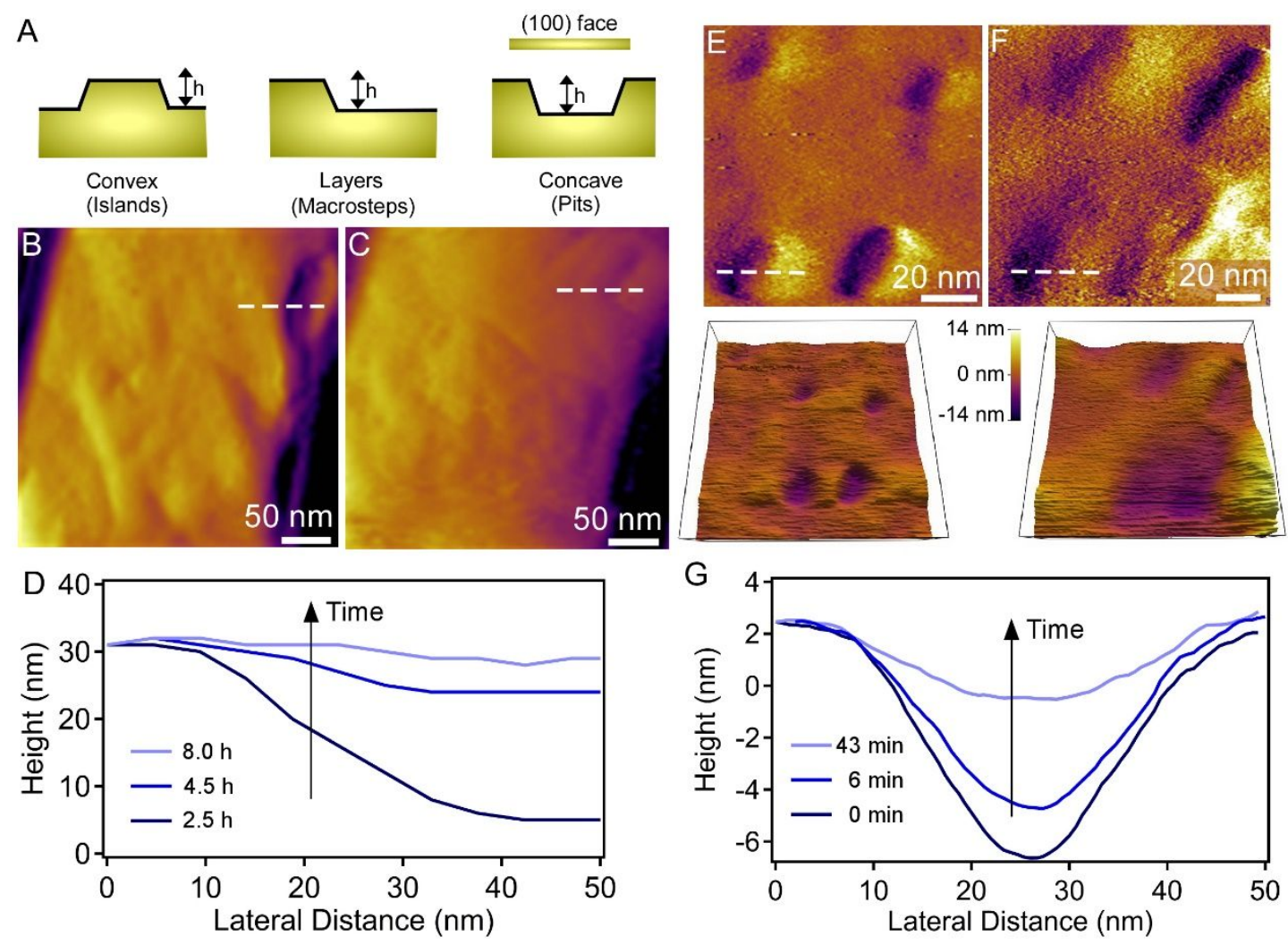

Figure 4. (A) Idealized schematics of three types of surface defects analyzed by in situ AFM: convex protrusions, macrosteps, and concave pits. (B and C) AFM amplitude mode images show the presence of (B) an initial macrostep (white dashed line) and (C) the same area after $8 \mathrm{~h}$ treatment with solution $\mathrm{S} 2$ at $25^{\circ} \mathrm{C}$. (D) Height profiles along the white dashed lines at various imaging times show a reduction in macrostep height. (E - G) Measurements of a separate USY crystal show the presence of etch pits on (E) an initial surface and (F) the same area after $1 \mathrm{~h}$ treatment with solution $\mathrm{S} 2$ at $25{ }^{\circ} \mathrm{C}$. Etch pits evolve from circular to ellipsoidal shapes with increasing treatment time. Corresponding 3-D height profiles are shown below each image. (G) Height profiles along the dashed lines in E and F showing the temporal reduction in etch pit height with increased AFM imaging time.

High resolution AFM images of USY crystal surfaces treated with surfactant for 60 min reveal a homogenous distribution of mesopores (Figure 5A and B). Quantitative analysis of mesopores over 
multiple crystal surfaces shows two distinct pore size distributions with an average pore size of $7 \mathrm{~nm}$. The height plot of a typical mesopore (Figure 5C) reveals a lateral dimension (pore diameter) of $7 \mathrm{~nm}$, whereas the depth of mesopores cannot be accurately determined by AFM owing to the relatively large curvature of the AFM tip; however, TEM images of ultramicrotomed samples (see Figure 7) reveal that mesopores are interconnected and penetrate deep into the crystals. It is also important to note that the lateral pore size distribution from AFM images is slightly higher than calculated from alternative techniques such as TEM and $\mathrm{N}_{2}$ physisorption (Figure 5D) due to the geometry of the AFM tip. However, the mesopore size distribution obtained by AFM is consistent with other techniques; and the same conclusion can be drawn when analyzing a sample after $6 \mathrm{~h}$ of treatment ex situ (Figure S6) where AFM images show a homogenous distribution of mesopores with similar pore sizes as those measured in situ.

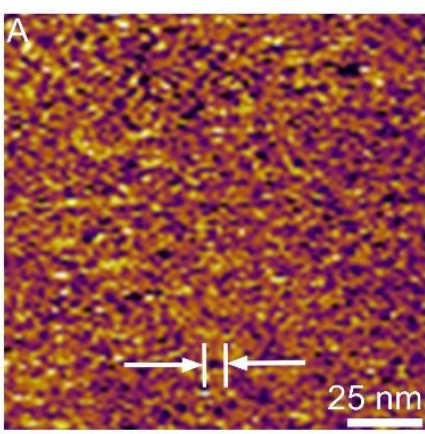

B

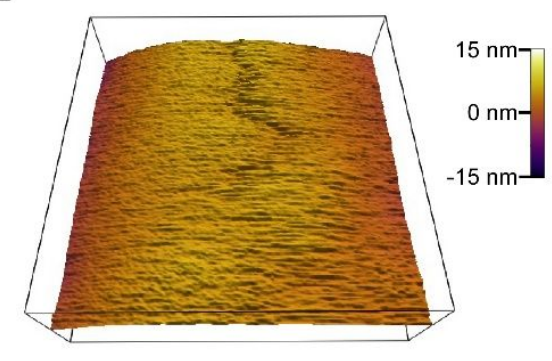

C

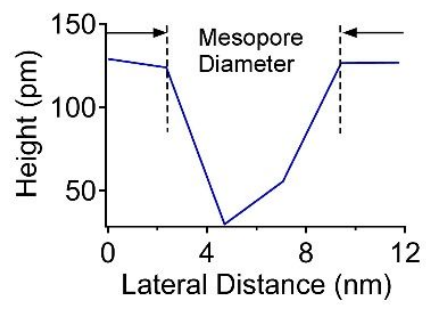

D

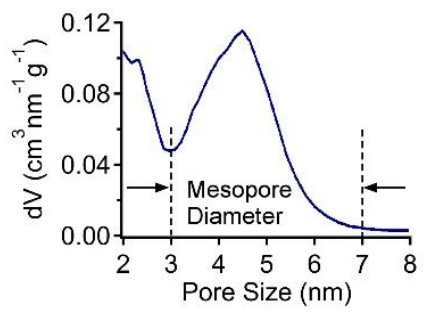

Figure 5. (A) High resolution AFM amplitude mode image and (B) corresponding 3-D rendering showing the uniform distribution of mesopores after 60 min of treatment in solution $\mathrm{S} 2$ at $25^{\circ} \mathrm{C}$ (see also Figure S6). (C) Height profile of the feature denoted in panel A (double white arrow) showing the 
diameter of a representative mesopore. (D) NL-DFT pore size distribution of mesostructured USY obtained from the adsorption branch of the $\mathrm{N}_{2}$ isotherms treated under similar conditions.

This AFM study provides important evidence for the processes of surfactant-mediated zeolite surface reconstruction to produce uniform mesopores. To confirm that this mesoporosity generation and crystal reconstruction also occur in the interior of the crystal, samples prepared ex situ under similar conditions were thoroughly characterized. The changes in the textural properties of the zeolite during the surfactant-templating process were evaluated by $\mathrm{N}_{2}$ physisorption (Figure 6A and Table S1) and TEM analyses (Figure 7) of solids prepared at different times. Additionally, milder (S1) and harsher (S3) alkaline solutions were used to investigate how the kinetics of mesopore formation depends upon the base concentration. In agreement to what has been observed by AFM, the parent USY zeolite is comprised of large mesopores as a result of steaming and acid treatment ${ }^{30}$ that contribute to the initial porosity, as can be deduced by the increasing amount of nitrogen adsorbed in the high relative pressure range $\left(P / P_{0}>0.8\right)$. Moreover, the isotherm of as received USY presents a hysteresis loop and some cavitation due to the presence of mesopores embedded in the zeolite that are only accessible through micropores. ${ }^{31}$ As the mesostructuring process develops, extracted solids display surfactant-templated mesopores from the first moments of treatment (Figure 6B), which increase in population with time until reaching a plateau (circles in Figure 6C). The mesoporosity generated by the surfactant-templating treatment evolves to generate very narrow pore size distributions with an average pore diameter ca. $4 \mathrm{~nm}$, as corresponds to the use of CTAB as a surfactant (Figure 6B). A second effect of surfactant-templating process, similar to what was has been previously reported for USY, ${ }^{19}$ is the disappearance of the larger porosity of the original zeolite, as evidenced by the plateau in the high relative pressure region of the $\mathrm{N}_{2}$ adsorption isotherms obtained at longer treatment times (triangles in Figure 6C). This is indicative of zeolite restructuring during the creation of 
new mesopores. ${ }^{18,19}$ The kinetics of the incorporation as well as the amount of mesoporosity introduced depends on the concentration of the base during the treatment. For instance, the mesoporosity of the zeolite increases by factors of 1.6, 2.3, and 3.5 in the first 30 min of treatment using solutions $\mathrm{S} 1(38 \mathrm{mM} \mathrm{NaOH}), \mathrm{S} 2(62.5 \mathrm{mM} \mathrm{NaOH})$, and $\mathrm{S} 3(94 \mathrm{mM} \mathrm{NaOH})$. It is worth noting that the incorporation of mesoporosity in CBV 780, under the conditions and time of treatment used in this study, has a negligible effect on the microporosity of these materials (see Figure S11). As shown in Figure $6 \mathrm{C}$, the evolution of the volume of micropores barely shows a very slight decrease in their microporosity with the time of treatment. 

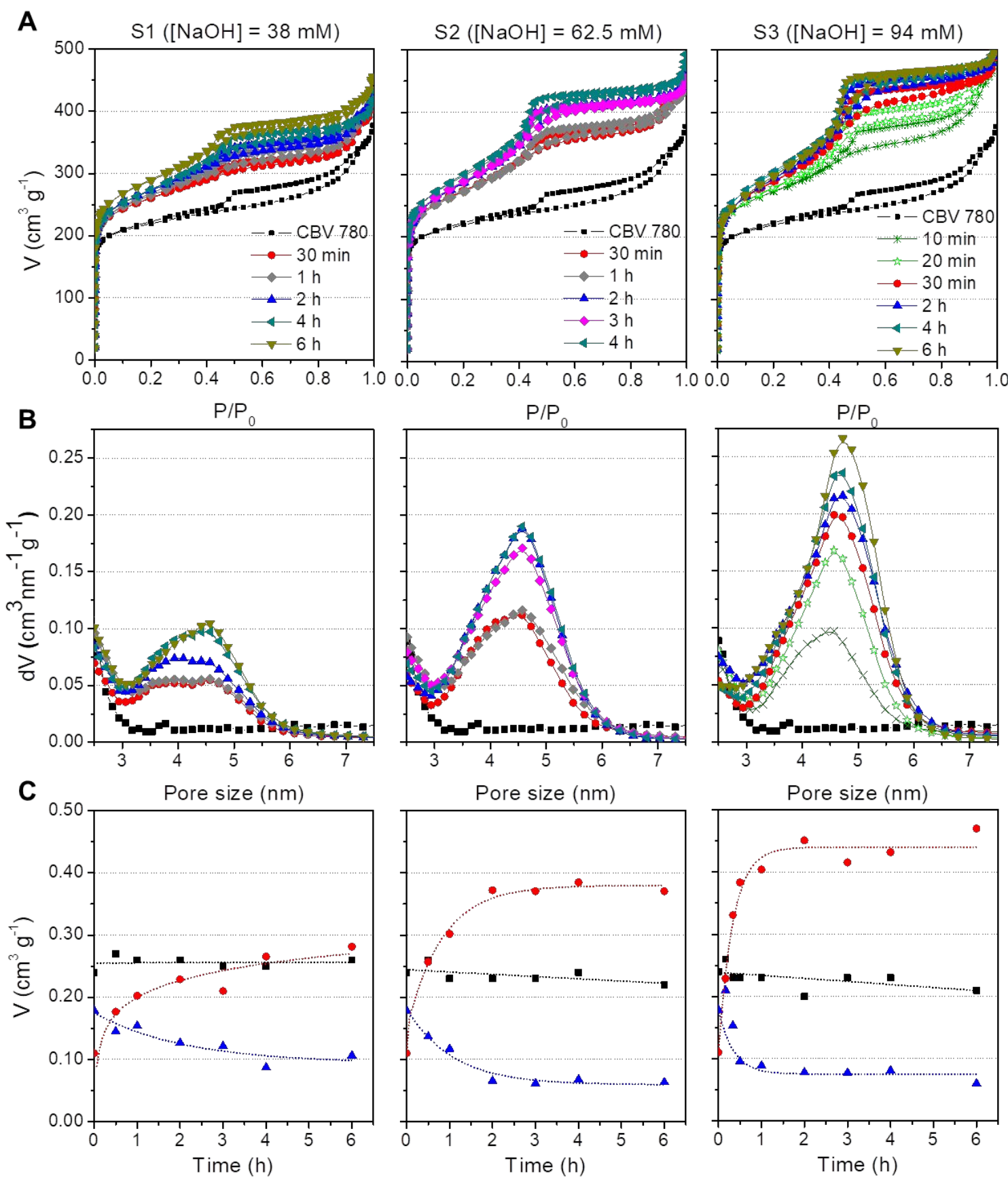

Figure 6. (A) $\mathrm{N}_{2}$ adsorption and desorption isotherms at $77 \mathrm{~K}$ for the ex situ surfactant-templated USY zeolites prepared using different base concentrations and treatment times at RT: 0 min (black), 30 min (red), $1 \mathrm{~h}$ (gray), $2 \mathrm{~h}$ (blue), $3 \mathrm{~h}$ (pink), $4 \mathrm{~h}$ (green), and $6 \mathrm{~h}$ (dark yellow). (B) NL-DFT pore size distribution of corresponding samples obtained from the adsorption branch of the $\mathrm{N}_{2}$ isotherms. (C) Changes in the volume of micropores (black square), surfactant-templated mesopores (red circle), and 


\begin{abstract}
large mesopores (blue triangle) for the different samples calculated using the NL-DFT method to the adsorption branch of the $\mathrm{N}_{2}$ isotherms.
\end{abstract}

Extensive TEM analysis of ultramicrotomed zeolites after different treatment times was carried out to further confirm the mesostructuring of entire crystals, in addition to surface restructuring observed by AFM. Statistical analysis of the development of mesoporosity was performed by studying at least 50 crystals of every sample (see Figures 7 and S12). TEM images of the parent zeolite shows the presence of large mesopores due to steaming (Figure 7A), which is consistent with the continuous uptake of $\mathrm{N}_{2}$ at $77 \mathrm{~K}$ at $\mathrm{P} / \mathrm{P}_{0}>0.8$ and the AFM analysis of the original USY sample. At early stages of treatment, two types of porosity can be observed in the TEM micrographs: the original large porosity and the uniform, smaller mesopores due to surfactant-templating (Figure 7B). As the mesostructuring evolves, the surfactant-templated mesopores develop in a homogenous manner through the whole crystal, as evidenced in micrographs after $2 \mathrm{~h}$ of treatment in solution S2 (Figure 7C), where no preferential sites for the development of mesoporosity can be observed. At longer treatment times, TEM images show the homogenous distribution of mesoporosity throughout the crystalline zeolites (Figure 7D-F). In agreement with $\mathrm{N}_{2}$ physisorption, TEM images of samples treated with solutions $\mathrm{S} 1, \mathrm{~S} 2$, and $\mathrm{S} 3$ reveal that the amount of mesoporosity incorporated depends on the alkalinity of the medium (Figure 7D, E, and $\mathrm{F}$, respectively). The disappearance of the larger initial porosity while the surfactant-templated mesoporosity develops is clearly observed in these images; however, the different kinetics shown by $\mathrm{N}_{2}$ physisorption are also observed by TEM. For samples treated with solutions S2 and S3 the large mesoporosity completely disappears after $6 \mathrm{~h}$ of treatment (see Figure 7E and F); and for the sample prepared with a lower base concentration (in solution S1), TEM images show only a fraction of the original large mesoporosity after the same time of treatment (Figure 7D). In all cases, the intracrystalline nature of the mesoporosity was confirmed by the crystal lattice observed at higher 
resolution (see for example Figure $7 \mathrm{G}$ ) and by digitally analyzing micrographs of ultramicrotomed samples. For example, we show in Figure 7G-I the TEM images and corresponding digital analyses (insets) of ultramicrotomed samples after $1 \mathrm{~h}$ of treatment with solution S2. The direct observation of mesoporosity (through the halo presented in the Fast Fourier Transformed (FFT) of each region) and crystallinity (responsible for the spots in the FFT) in the ultramicrotomed slices of surfactant-templated zeolites is strong evidence for the presence intracrystalline mesoporosity in the interior of the zeolite crystals. These results obtained for CBV 780 are consistent with our previously reported work about the intracrystalline nature of the mesoporosity introduced in CBV 720 using the same method, which has been proved by a combination of techniques, including electron tomography (ET). ${ }^{16,18,19}$
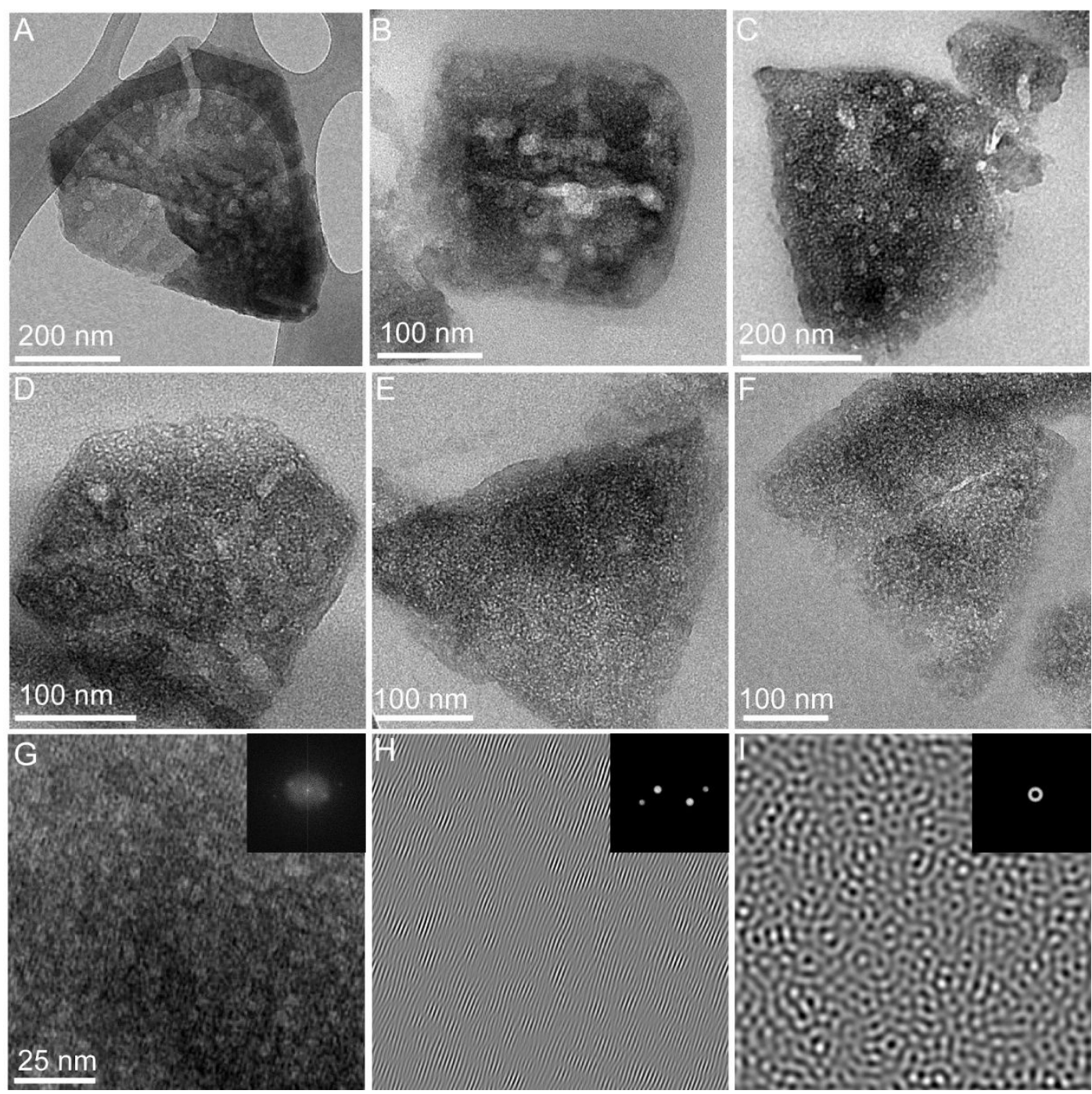
Figure 7. TEM images of ultramicrotomed samples for (A) as received USY (CBV 780) and surfactant-templated zeolites treated in solution S2 for the following times: (B) 30 min and (C) $2 \mathrm{~h}$. Representative images are shown for samples treated for $6 \mathrm{~h}$ in different alkaline solutions: (D) S1, (E) S2, and (F) S3. (G) TEM micrograph of an ultramicrotomed slice of a sample treated for $1 \mathrm{~h}$ using solution S2. The inset shows its corresponding FFT. (H) Reconstruction of the crystalline phase by FFT of the spots. (I) Reconstruction of the features presenting the identical mesopores distance by FFT on the halo.

It is important to note that the mesostructuring of zeolite USY does not involve the filling of its large pre-existing mesopores. First, we have previously demonstrated that zeolites without initial mesoporosity, such as $\mathrm{NaY}$, can be mesostructured with surfactants. ${ }^{23}$ Second, the volume of mesoporosity introduced can be significantly higher than the pre-existent porosity (i.e. $0.29 \mathrm{~cm}^{3} \mathrm{~g}^{-1}$ initially compared to $0.45 \mathrm{~cm}^{3} \mathrm{~g}^{-1}$ after surfactant-templating treatment with solution S2); and third, the surfactant-templated mesoporosity introduced with surfactant occurs throughout the entire crystal, as evidenced by extensive TEM studies (Figure 7). The mesostructuring process can be incomplete with insufficient treatment time, or a low quantity of $\mathrm{NaOH}$ is used. In partially mesostructured zeolites, the unconverted areas are zeolitic (microporous) domains, as recently described by Galarneau and coworkers. ${ }^{32}$ On the other hand, if the treatment is excessive (e.g. high $\mathrm{pH}$ ), a fraction of the zeolite can be dissolved and an amorphous mesoporous phase is formed (Figure S13), as reported by García-Martínez and coworkers ${ }^{4}$ and recently confirmed independently. ${ }^{32}$ Therefore, it is critically important to judiciously select the mesostructuring conditions to produce high quality surfactanttemplated zeolites and to avoid the formation of spurious phases. For severely steamed zeolites (e.g. CBV 780) it is also important to mention that these samples contain some heterogeneity in composition as not all the crystals are equally dealuminated during the preparation of USY. The population of 
crystals with lower aluminum content are easier to treat by the surfactant-templating process and, consequently, show a higher degree of mesoporosity (Figure S14).

The preservation of the crystalline structure of the zeolites after the mesostructuring process was confirmed by X-ray diffraction (Figure S9). The diffraction patterns of parent USY and the highly mesoporous sample treated for $6 \mathrm{~h}$ with solution S2 show intense peaks associated to the FAU structure even after the incorporation of a large amount of mesoporosity $\left(0.37 \mathrm{~cm}^{3} \mathrm{~g}^{-1}\right)$. However, if the same treatment is performed to USY zeolite in the absence of CTAB (i.e. using only alkaline $\mathrm{NaOH}$ solutions), the crystalline structure of the zeolite is totally lost and the resultant powder XRD patterns indicate an amorphous product, which confirms that CTAB adds a level of protection that prevents a crystal-to-amorphous transformation. ${ }^{18}$

Additional evidence of crystal reconstruction during surfactant-templating process was obtained by ${ }^{27} \mathrm{Al}$ NMR and elemental analysis (ICP). The NMR spectrum of the original USY sample (Figure 8) shows two bands around 60 and 0 ppm that correspond to Al in tetrahedral (Al(IV)) and octahedral (Al(VI)) coordination environments, respectively. ${ }^{33,} 34$ The band at around 0 ppm disappears completely during the treatment (even for short treatment times), indicating the elimination of the octahedral Al (i.e. extra-framework species). Interestingly, ICP measurements of extracted reaction liquids after various treatment times do not show any leaching of $\mathrm{Al}$, thereby suggesting that the removal of octahedral Al leads to its the re-insertion of extra-framework Al into the zeolite framework (as it has been observed in different studies for zeolites treated with alkaline solutions). ${ }^{35-37}$ Moreover, deconvolution of the peak associated with tetrahedral Al further results in two contributions, Al(IV)a and $\mathrm{Al}(\mathrm{IV}) \mathrm{b}$. The Al(IV)a species is assigned to tetra-coordinated Al atoms in the framework (chargebalanced by protons), ${ }^{34}$ whereas the attribution of $\mathrm{Al}(\mathrm{IV}) \mathrm{b}$ is more challenging. In zeolites, this band can be due to distorted tetra-coordinated $\mathrm{Al} \operatorname{species}^{38}$ or to framework $\mathrm{Al}$ atoms interacting with cationic species present outside the network. ${ }^{39}$ On the basis that surfactant-templating treatment causes 
a slight increase of the $\mathrm{Al}(\mathrm{IV}) \mathrm{b}$ contribution, we hypothesize this signal is related to the interaction of the framework $\mathrm{Al}$ with $\mathrm{CTA}^{+}$cations and to the subsequent distortion of the tetrahedral coordination.

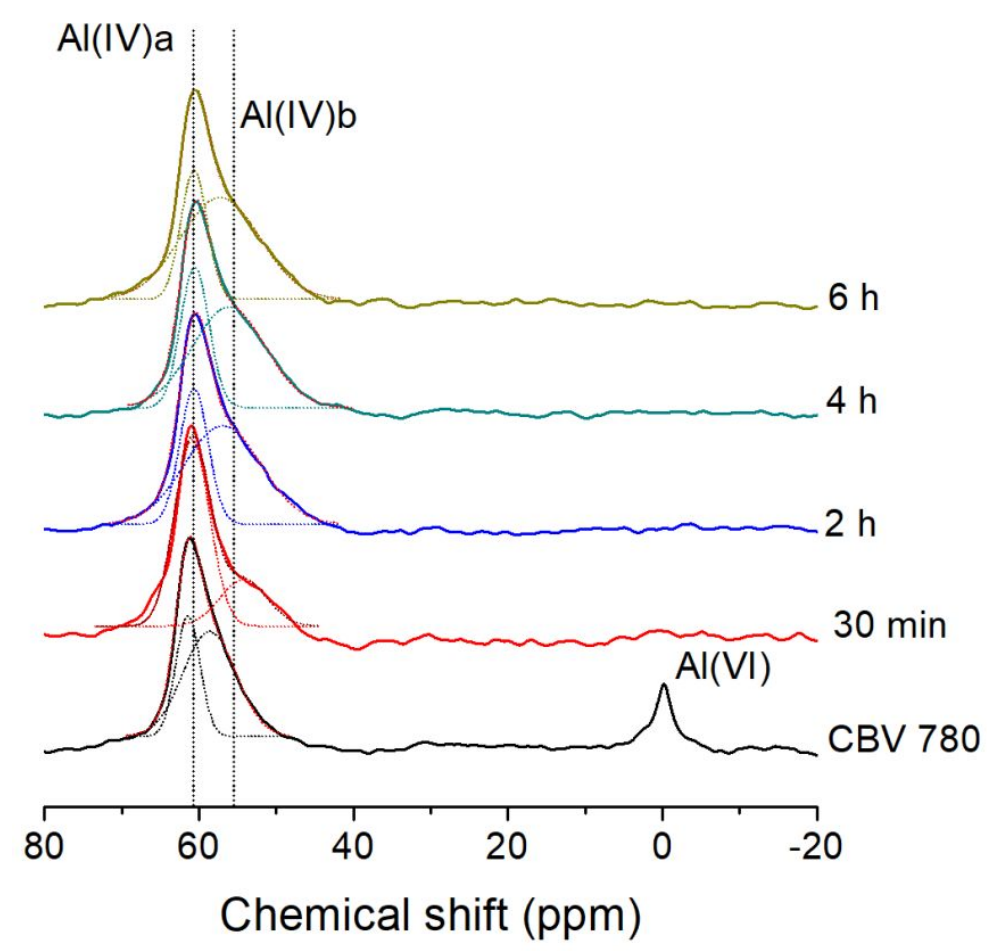

Figure 8. ${ }^{27} \mathrm{Al}$ NMR spectra of the parent USY zeolite (CBV 780) and surfactant-templated zeolites treated with solution S2 and different reaction times spanning from $30 \mathrm{~min}$ to $6 \mathrm{~h}$. The dotted lines show the deconvolution of the individual bands.

\section{CONCLUSIONS}

In summary, we have been able to directly observe the development of mesoporosity in USY through surfactant-templating by the use of AFM. In a time-resolved manner, we have observed how the defects present in USY, which are produced by the supplier by a combination of steaming and acid washes, are removed while narrower surfactant-templated mesoporosity develops. Interestingly, these processes occur rapidly at room temperature to produce a final product with uniformly-distributed mesopores throughout the entire crystal. Molecular level details of the crystal reconstruction during 
surfactant-templating are not fully understood. Nevertheless, this study provides additional evidence that zeolite crystals do not dissolve to produce mesoporosity. Indeed, elemental analysis of the liquids post-treatment reveals the negligible presence of soluble species, while ca. $100 \%$ of the solids are recovered at any given time of the surfactant-templating process. Extensive in situ microscopy confirmed that zeolite USY does not dissolve during the surfactant-templating treatment, although short-scale breaking and reconstruction of the zeolite framework is necessary for the generation of intracrystalline mesoporosity. The use of additional techniques such as $\mathrm{N}_{2}$ adsorption, TEM of ultramicrotomed samples, powder XRD, and ${ }^{27} \mathrm{Al}$ NMR have confirmed that the surface phenomena observed by AFM is consistent with changes occurring throughout the entire crystal. Collectively, these techniques provide new and unequivocal insights that confirm surfactant templating is a facile and efficient method to induce reconstruction of the crystal, the healing of defects, the reinsertion of extraframework Al, and the disappearance of large mesoporosity towards the generation of optimal FAUtype zeolites for catalytic applications.

\section{Supporting Information}

Supplementary Information accompanies this paper at http://pubs.acs.org: characterization of the samples by AFM images, TEM analyses, powder XRD, physisorption and movies showing the evolution of the samples during the in situ AFM experiments.

\section{Acknowledgments}

N.L. acknowledges funding from the University of Alicante, through the "Programa de retención y captación de talento" (ref. UATALENTO17-05). J.D.R. acknowledges funding from the Welch Foundation (Award E-1794).

\section{Funding}

University of Alicante, ref. UATALENTO17-05. 
Welch Foundation, Award E-1794.

\section{References}

1. Kresge, C. T.; Roth, W. J., The discovery of mesoporous molecular sieves from the twenty year perspective. Chemical Society Reviews 2013, 42, (9), 3663-3670.

2. Kresge, C.; Leonowicz, M.; Roth, W. J.; Vartuli, J.; Beck, J., Ordered mesoporous molecular sieves synthesized by a liquid-crystal template mechanism. Nature 1992, 359, (6397), 710.

3. Perego, C.; Millini, R., Porous materials in catalysis: challenges for mesoporous materials. Chemical Society Reviews 2013, 42, (9), 3956-3976.

4. Sachse, A.; Garcia-Martinez, J., Surfactant-templating of zeolites: from design to application. Chemistry of Materials 2017, 29, (9), 3827-3853.

5. Rimer, J. D.; Chawla, A.; Le, T. T., Crystal Engineering for Catalysis. Annual Review of Chemical and Biomolecular Engineering 2018, 9, (1), 283-309.

6. $\quad$ Prasomsri, T.; Jiao, W.; Weng, S. Z.; Martinez, J. G., Mesostructured zeolites: bridging the gap between zeolites and MCM-41. Chemical Communications 2015, 51, (43), 8900-8911.

7. Davis, M. E., Mesoporous zeolites: preparation, characterization and applications. John Wiley \& Sons: 2015.

8. Pérez-Ramírez, J.; Christensen, C. H.; Egeblad, K.; Christensen, C. H.; Groen, J. C., Hierarchical zeolites: enhanced utilisation of microporous crystals in catalysis by advances in materials design. Chemical Society Reviews 2008, 37, (11), 2530-2542.

9. Roth, W. J.; Nachtigall, P.; Morris, R. E.; Cejka, J., Two-dimensional zeolites: current status and perspectives. Chemical reviews 2014, 114, (9), 4807-4837.

10. Serrano, D.; Pizarro, P., Synthesis strategies in the search for hierarchical zeolites. Chemical Society Reviews 2013, 42, (9), 4004-4035.

11. Chawla, A.; Li, R.; Jain, R.; Clark, R. J.; Sutjianto, J. G.; Palmer, J. C.; Rimer, J. D., Cooperative effects of inorganic and organic structure-directing agents in ZSM-5 crystallization. Molecular Systems Design \& Engineering 2018, 3, (1), 159-170.

12. Ying, J. Y.; Martinez, J. G., Mesostructured zeolitic materials, and methods of making and using the same. US Patent, No. 7589041 B2 Sept. 15, 2009.

13. Li, K.; Valla, J.; Garcia - Martinez, J., Realizing the commercial potential of hierarchical zeolites: new opportunities in catalytic cracking. ChemCatChem 2014, 6, (1), 46-66.

14. García-Martínez, J.; Johnson, M.; Valla, J.; Li, K.; Ying, J. Y., Mesostructured zeolite Y-high hydrothermal stability and superior FCC catalytic performance. Catalysis Science \& Technology 2012, 2, (5), 987-994.

15. García-Martínez, J.; Li, K.; Krishnaiah, G., A mesostructured Y zeolite as a superior FCC catalyst-from lab to refinery. Chemical Communications 2012, 48, (97), 11841-11843.

16. Garcia - Martinez, J.; Xiao, C.; Cychosz, K. A.; Li, K.; Wan, W.; Zou, X.; Thommes, M., Evidence of intracrystalline mesostructured porosity in zeolites by advanced gas sorption, electron tomography and rotation electron diffraction. ChemCatChem 2014, 6, (11), 3110-3115.

17. Thomas, J. M.; Leary, R. K., A Major Advance in Characterizing Nanoporous Solids Using a Complementary Triad of Existing Techniques. Angewandte Chemie International Edition 2014, 53, (45), 12020-12021.

18. Sachse, A.; Grau-Atienza, A.; Jardim, E. O.; Linares, N.; Thommes, M.; Garcia-Martinez, J., Development of intracrystalline mesoporosity in zeolites through surfactant-templating. Crystal Growth \& Design 2017, 17, (8), 4289-4305. 
19. Linares, N.; Sachse, A.; Serrano, E.; Grau-Atienza, A.; De Oliveira Jardim, E.; SilvestreAlbero, J.; Cordeiro, M. A. L.; Fauth, F.; Beobide, G.; Castillo, O.; García-Martínez, J., In Situ TimeResolved Observation of the Development of Intracrystalline Mesoporosity in USY Zeolite. Chemistry of Materials 2016, 28, (24), 8971-8979.

20. Linares, N.; Jardim, E. O.; Sachse, A.; Serrano, E.; García - Martínez, J., The Energetics of Surfactant - Templating of Zeolites. Angewandte Chemie International Edition 2018, 57, (28), 87248728.

21. Kumar, M.; Choudhary, M. K.; Rimer, J. D., Transient modes of zeolite surface growth from 3D gel-like islands to 2D single layers. Nature communications 2018, 9, (1), 2129.

22. Shete, M.; Kumar, M.; Kim, D.; Rangnekar, N.; Xu, D.; Topuz, B.; Agrawal, K. V.; Karapetrova, E.; Stottrup, B.; Al-Thabaiti, S.; Basahel, S.; Narasimharao, K.; Rimer, J. D.; Tsapatsis, M., Nanoscale Control of Homoepitaxial Growth on a Two-Dimensional Zeolite. Angewandte Chemie International Edition 2017, 56, (2), 535-539.

23. Lupulescu, A. I.; Rimer, J. D., In situ imaging of silicalite-1 surface growth reveals the mechanism of crystallization. Science 2014, 344, (6185), 729-732.

24. Brent, R.; Anderson, M. W., Fundamental Crystal Growth Mechanism in Zeolite L Revealed by Atomic Force Microscopy. Angewandte Chemie International Edition 2008, 47, (29), 5327-5330.

25. Cubillas, P.; Castro, M.; Jelfs, K. E.; Lobo, A. J. W.; Slater, B.; Lewis, D. W.; Wright, P. A.; Stevens, S. M.; Anderson, M. W., Spiral Growth on Nanoporous Silicoaluminophosphate STA-7 as Observed by Atomic Force Microscopy. Crystal Growth \& Design 2009, 9, (9), 4041-4050.

26. Anderson, M. W.; Gebbie-Rayet, J. T.; Hill, A. R.; Farida, N.; Attfield, M. P.; Cubillas, P.; Blatov, V. A.; Proserpio, D. M.; Akporiaye, D.; Arstad, B.; Gale, J. D., Predicting crystal growth via a unified kinetic three-dimensional partition model. Nature 2017, 544, 456.

27. Kumar, M.; Luo, H.; Román-Leshkov, Y.; Rimer, J. D., SSZ-13 crystallization by particle attachment and deterministic pathways to crystal size control. Journal of the American Chemical Society 2015, 137, (40), 13007-13017.

28. Mandemaker, L. D. B.; Filez, M.; Delen, G.; Tan, H.; Zhang, X.; Lohse, D.; Weckhuysen, B. M., Time-Resolved In Situ Liquid-Phase Atomic Force Microscopy and Infrared Nanospectroscopy during the Formation of Metal-Organic Framework Thin Films. The Journal of Physical Chemistry Letters 2018, 9, (8), 1838-1844.

29. Cychosz, K. A.; Guillet-Nicolas, R.; Garcia-Martinez, J.; Thommes, M., Recent advances in the textural characterization of hierarchically structured nanoporous materials. Chemical Society Reviews 2017, 46, (2), 389-414.

30. Kenvin, J.; Mitchell, S.; Sterling, M.; Warringham, R.; Keller, T. C.; Crivelli, P.; Jagiello, J.; Pérez - Ramírez, J., Quantifying the complex pore architecture of hierarchical faujasite zeolites and the impact on diffusion. Advanced Functional Materials 2016, 26, (31), 5621-5630.

31. Thommes, M.; Kaneko, K.; Neimark, A. V.; Olivier, J. P.; Rodriguez-Reinoso, F.; Rouquerol, J.; Sing, K. S., Physisorption of gases, with special reference to the evaluation of surface area and pore size distribution (IUPAC Technical Report). Pure and Applied Chemistry 2015, 87, (9-10), 1051-1069.

32. Mehlhorn, D.; Rodriguez, J.; Cacciaguerra, T.; Andrei, R.-D.; Cammarano, C.; Guenneau, F.; Gedeon, A.; Coasne, B.; Thommes, M.; Minoux, D.; Aquino, C.; Dath, J.-P.; Fajula, F.; Galarneau, A., Revelation on the Complex Nature of Mesoporous Hierarchical FAU-Y Zeolites. Langmuir 2018, 34, (38), 11414-11423.

33. Agudelo, J.; Hensen, E.; Giraldo, S.; Hoyos, L., Influence of steam-calcination and acid leaching treatment on the VGO hydrocracking performance of faujasite zeolite. Fuel Processing Technology 2015, 133, 89-96. 
34. Agudelo, J.; Mezari, B.; Hensen, E.; Giraldo, S.; Hoyos, L., On the effect of EDTA treatment on the acidic properties of USY zeolite and its performance in vacuum gas oil hydrocracking. Applied Catalysis A: General 2014, 488, 219-230.

35. Zhang, Z.; Liu, X.; Xu, Y.; Xu, R., Realumination of dealuminated zeolites Y. Zeolites 1991, $11,(3), 232-238$.

36. Calsavara, V.; Sousa-Aguiar, E. F.; Machado, N. R. F., Reactivity of USY extraframework alumina in alkaline medium. Zeolites 1996, 17, (4), 340-345.

37. Menezes, S.; Camorim, V.; Lam, Y.; San Gil, R.; Bailly, A.; Amoureux, J., Characterization of extra-framework species of steamed and acid washed faujasite by MQMAS NMR and IR measurements. Applied Catalysis A: General 2001, 207, (1-2), 367-377.

38. Katada, N.; Nakata, S.; Kato, S.; Kanehashi, K.; Saito, K.; Niwa, M., Detection of active sites for paraffin cracking on USY zeolite by 27Al MQMAS NMR operated at high magnetic field $16 \mathrm{~T}$. Journal of Molecular Catalysis A: Chemical 2005, 236, (1-2), 239-245.

39. Malicki, N.; Mali, G.; Quoineaud, A.-A.; Bourges, P.; Simon, L. J.; Thibault-Starzyk, F.; Fernandez, C., Aluminium triplets in dealuminated zeolites detected by 27Al NMR correlation spectroscopy. Microporous and Mesoporous Materials 2010, 129, (1-2), 100-105. 